Pacific Journal of Mathematic 


\title{
INTEGRAL EQUIVALENCE OF VECTORS OVER LOCAL MODULAR LATTICES, II
}

\author{
JoHN S. HSIA
}

In an earlier paper in this Journal we have shown that the integral equivalence problem for vectors in a modular lattice $L$ on a dyadic local field $F$ can be determined, for $\operatorname{dim} L \neq 4$, 5,6 , by inspecting the numbers represented in $F$ by the characteristic sets which are canonically associated to the given vectors. The purpose of this paper is to remove this dimensional restriction of $L$. In addition, we shall discuss the effective determination of integral equivalences amongst vectors as well as derive some "cancellation" results. Finally, we prove, as expected, that this same improvement carries over in the characteristic two situation.

The presentation of the results contatined herein shall be as follows:

1. Preliminary observations.

2. Statement and proof of the main theorem.

3. Effective computability.

4. Cancellation theorems.

5. Characteristic two case.

We shall adhere to the same terminology and notations as those contained in [2]. The following data will be fixed throughout this paper. $L$ is a unimodular lattice, $u$ and $v$ are two maximal (primitive) vectors in $L$ having the same quadratic length $Q(u)=Q(v)=\delta$. Integral equivalence between $u$ and $v$ shall always be denoted by $u \sim v$.

1. Preliminary observations. For any maximal vector $w \in L$, the characteristic set of $w$ in $L$ is defined as

$$
\mathfrak{M}_{w}=\{x \in L \mid B(x, w)=1\} \text {. }
$$

It is easy to see that

$$
\mathfrak{M}_{w}=\hat{w}+\langle w\rangle^{\perp}=\left\{\hat{w}+y \mid y \in\langle w\rangle^{\perp}\right\}
$$

where $\hat{w}$ is any vector in $\mathfrak{M}_{w}$.

Notation 1.1. Almost always when we write

$$
\mathcal{O} x+\mathcal{O} y \cong A(\alpha, \beta)
$$

we mean that 


$$
Q(x)=\alpha, \quad Q(y)=\beta, \quad B(x, y)=1 .
$$

However, sometimes-and the context under which it occurs will be clear - it may simply mean that the lattice $\mathscr{O} x+\mathcal{O} y$ is isometric to $A(\alpha, \beta)$ without necessarily implying that the basis vectors $\{x, y\}$ satisfy $(*)$.

Lemma 1.2. $\langle u\rangle^{\perp} \cong\langle v\rangle^{\perp}$ does not, in general, imply $u \sim v$.

Proof. Let $L=\mathcal{O} x+\mathcal{O} u=A\left(a^{\prime}, 0\right)$ where $a^{\prime}$ is a norm generator for $\mathscr{C} L$. Suppose $v=\varepsilon\left[u-\left(2 / \alpha^{\prime}\right) x\right]$ for some unit $\varepsilon$, and $y=-\varepsilon^{-1} x$. Then,

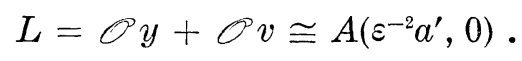

Clearly then,

$$
\langle u\rangle^{\perp}=\varnothing u \cong \mathscr{O} v=\langle v\rangle^{\llcorner} .
$$

Any isometry $\sigma \in O(L)$ sending $u$ onto $v$ takes $x$ onto, say,

$$
\sigma(x)=\alpha y+\beta v, \alpha, \beta \in \mathcal{O} .
$$

But, $B(\sigma(x), v)=B(x, u)=1$ implies $\alpha=1$. The length of $\sigma(x)$ must, on the other hand, be $a^{\prime}$ so that

$$
a^{\prime}\left(\frac{1-\varepsilon^{2}}{\varepsilon^{2}}\right)+2 \beta=0 .
$$

This equation (**), of course, does not always admit integral solution for $\beta$ when norm generator $a^{\prime}$ and unit $\varepsilon$ can be arbitrary. Thus, we can not expect, in general, to have $u \sim v$ with just requiring their orthogonal complements to be isometric.

1.3. Unless otherwise specified, $\operatorname{dim} L \geqq 4$ shall be assumed throughout the rest of this paper. To avoid excessive repetitions, let us fix a few more notations here. For any primitive vector $w \in L$, and any vector $\bar{w} \in \mathfrak{M}_{*}$, put

$$
L(w ; \bar{w})=\varnothing w+\varnothing \bar{w} ; \quad M(w ; \bar{w})=L(w ; \bar{w})^{-} .
$$

LEMMA 1.4. Let $\operatorname{dim} L$ be arbitrary. $Q\left(\mathfrak{M}_{u}\right)=Q\left(\mathfrak{M}_{v}\right)$ implies $\langle u\rangle^{\perp} \cong\langle v\rangle^{\perp}$.

Proof. Pick $\bar{u} \in \mathfrak{M}_{u}$, and $\bar{v} \in \mathfrak{M}_{v}$ such that $Q(\bar{u})=Q(\bar{v})$. Let $D$ be the common discriminant of $L(u ; \bar{u})$ and $L(v ; \bar{v})$. Then, 


$$
\begin{aligned}
& \langle u\rangle^{\perp}=M(u ; \bar{u}) \perp \mathcal{O}\langle u-\delta \bar{u}\rangle \cong M(u ; \bar{u}) \perp\langle\delta D\rangle \\
& \langle v\rangle^{\perp}=M(v ; \bar{v}) \perp \mathcal{O}\langle v-\delta \bar{v}\rangle \cong M(v ; \bar{v}) \perp\langle\delta D\rangle .
\end{aligned}
$$

An application of Witt's theorem yields

$$
F\langle u\rangle^{\perp} \cong F\langle v\rangle^{\perp} \text {. }
$$

Also, it is not difficult to see that hypothesis of the lemma implies the equality of the norm groups (via 93:21, [4]) may be assumed:

$$
\mathscr{G} M(u ; \bar{u})=\mathscr{G} M(v ; \bar{v})=\mathscr{G}^{*}
$$

when $\operatorname{dim} L \geqq 5$; here, $\mathscr{G}^{*}=\mathscr{G}\left(\langle u\rangle^{\perp}\right)=\mathscr{G}\left(\langle v\rangle^{\perp}\right)$. Hence, by O'Meara's theorem on modular lattices $(93: 16,[4]) M(u ; \bar{u})$ is isometric to $M(v ; \bar{v})$. So let $\operatorname{dim} L=4$. Adjoin the hyperbolic lattice $A(0,0)$ to $L$ and call the enlarged lattice $L^{\prime}$. Then, $\langle u\rangle^{\perp}$ is isometric to $\langle v\rangle^{\perp}$ in $L^{\prime}$. But,

$$
\langle u\rangle^{\perp}\left(\text { in } L^{\prime}\right) \cong\langle u\rangle^{\lrcorner}(\text {in } L) \perp A(0,0),
$$

and similarly for $\langle v\rangle^{\prime}$. Cancelling $A(0,0)$ gives the desired result. When $\operatorname{dim} L \leqq 3$, the proof is entirely trivial.

REMARK 1.5. The proof of Lemma 1.4 is one without using the fact that $Q\left(\mathfrak{M}_{u}\right)$ equals $Q\left(\mathfrak{M}_{v}\right)$ implies $u \sim v$ for large enough dimension of $L$ as we did in Corollary 4.2, [2].

LEMMA 1.6. If $|\delta|=0,1$, then $Q\left(\mathfrak{M}_{u}\right)=Q\left(\mathfrak{M}_{v}\right)$ implies $u \sim v$.

Proof. By Lemma 1.4, $\langle u\rangle^{\perp} \cong\langle v\rangle^{\perp}$. If $\delta$ is an unit, then everything is clear. Otherwise, let $\delta=0$. Let $\bar{u}$ and $\bar{v}$ be the two vectors as in Lemma 1.4, then we have the radical splittings

$$
\begin{aligned}
& \langle u\rangle^{\perp}=\operatorname{Rad}\langle u\rangle^{\perp} \perp M(u ; \bar{u})=\mathcal{O} u \perp M(u ; \bar{u}) \\
& \langle v\rangle^{\perp}=\operatorname{Rad}\langle v\rangle^{\perp} \perp M(v ; \bar{v})=\mathcal{O} v \perp M(v ; \bar{v}) .
\end{aligned}
$$

But, in this case

$$
\langle u\rangle^{\perp} \cong\langle v\rangle^{\perp} \text { if and only if } M(u ; \bar{u}) \cong M(v ; \bar{v}) .
$$

In view of this Lemma 1.6, we shall henceforth, unless otherwise noted, assume that $|\delta|$ is neither 0 nor 1 .

1.7. In the proof of Theorem 4.4, [2], an important fact used was Lemma 4.5 , [2], whose proof can be much simplified by observing that in the case when both $u$ and $v$ are Type I vectors, $Q\left(\mathfrak{M}_{u}\right)$ equals $Q\left(\mathfrak{M}_{v}\right)$ implies $u$ and $v$ are of the same parity, and also $\langle u\rangle^{\perp}$ is isometric to $\langle v\rangle^{\perp}$ via Lemma 1.4. Therefore, $u \sim v$ by Proposition 3.5, 
[2]. Hence, Lemma 4.5, [2] becomes easy to see.

\section{Statement and proof of the main theorem.}

MAIN TheOREm 2.1. Let $L$ be an unimodular lattice over a dyadic local field; then, two maximal (primitive) vectors in $L$ having the same quadratic length are integrally equivalent if and only if their characteristic sets represent the same numbers in the field.

Notations 2.2. Put $\mathscr{G}^{*}=\mathscr{G}\left(\langle u\rangle^{\perp}\right)=\mathscr{G}\left(\langle v\rangle^{\perp}\right)$. For any $\bar{u} \in \mathfrak{M}_{u}$, we set

$$
\lambda_{\bar{u}}=\delta Q(\bar{u})-1,
$$

the discriminant of $\mathscr{O} \bar{u}+\mathcal{O} u$. The letters $a, b$ shall always be used for norm and weight (base) generator of $\mathscr{G} L$ respectively; similarly, $a^{*}$ and $b^{*}$ for the norm group $\mathscr{G}^{*}$.

2.3. In view of Theorem 4.1, [2], it suffices to prove for $\operatorname{dim} L=$ 4, 5,6. Proposition 3.5, [2] allows us to assume that both $u$ and $v$ are Type II vectors.

2.4. Because $u$ and $v$ are Type II vectors, we may further suppose henceforth that $\langle u\rangle^{\perp}$ (hence, also $\langle v\rangle^{\perp}$ ) are not "depleted" in the sense of [1]. For, if not, then the norm group of $M(u ; \bar{u})$ will be equal to $\mathscr{G}^{*}, \forall \bar{u} \in \mathfrak{M}_{u}$. Consequently, the integral equivalence between $u$ and $v$ may be readily deduced from the hypothesis of the Main Theorem. So, in particular, $a^{*} b^{*} \sim \pi$ (i.e., ord $a^{*}+\operatorname{ord} b^{*}$ is odd).

2.5. Roughly, we first observe that the number $\delta$ may be assumed to have a special feature. Using this "reduction lemma," we settle the 4-dimensional case by computational means; in the case of $\operatorname{dim} L$ equals five, we shall show that the hypothesis, and hence also the conclusion, of Theorem 4.4, [2] is satisfied. Finally, the $\operatorname{dim} L=6$ case falls through by a modified argument tailored after the 5-dimensional situation.

Lemma 2.6. If the quadratic defect $\mathscr{D}\left(\delta \lambda_{\bar{u}} a^{*}\right)$ is strictly contained in the ideal $a^{*} b^{*} \mathscr{O}$ for some norm generator $a^{*}$ of $\mathscr{G}^{*}$, then $\mathscr{D}\left(\delta \lambda_{u} a^{\prime}\right) \varsubsetneqq a^{*} b^{*} \mathcal{O}$ for every norm generator $a^{\prime}$ of $\mathscr{G}^{*}$.

Proof. Put $\delta \lambda_{u}=a^{*} t^{2}+b^{*} t^{2}+b^{*} \alpha ; t, \alpha \in \mathcal{O}$. Now, $u$ is a Type II vector implies $|t|<1$. The hypothesis together with the fact that $a^{*} b^{*} \sim \pi$ yields $|\alpha|<1$. Now then, 


$$
a^{\prime}=a^{*} \varepsilon^{2}+b^{*} M,|\varepsilon|=1, M \in \mathcal{O}
$$

and

$$
\delta \lambda_{\bar{u}} a^{\prime}=a^{* 2} t^{2} \varepsilon^{2}+a^{*} b^{*} \alpha \varepsilon^{2}+a^{*} t^{2} b^{*} M+b^{* 2} \alpha M .
$$

The quadratic defect of the right hand side of $(0)$ is clearly contained in a $a^{*} b^{*} \mathcal{O}$ since each of the last three terms is in it.

CoROLlaRY 2.7. If $\exists \bar{u} \in \mathfrak{M}_{u}$ such that for some $a^{*}$, we have $\mathscr{D}\left(\delta \lambda_{\bar{u}} a^{*}\right)=a^{*} b^{*} \mathcal{O}$ then, $\mathscr{D}\left(\delta \lambda_{\bar{u}} a^{\prime}\right)=a^{*} b^{*} \mathcal{O}$ for every norm generator $a^{\prime}$ of $\mathscr{Q}^{*}$.

CoRollary 2.8. If $\exists \bar{u} \in M_{u}$ such that for some $a^{*}$, we have $\mathscr{D}\left(\delta \lambda_{\bar{u}} a^{*}\right) \varsubsetneqq a^{*} b^{*} \mathcal{O}$, then for every $x \in \mathfrak{M}_{u}$ such that $\lambda_{\bar{u}} \in \lambda_{x} \mathscr{C}^{2}(\mathscr{C}$ denotes the group of units in $\mathcal{O}^{\circ}$, the inequality below is valid

$$
\mathscr{D}\left(\delta \lambda_{x} a^{\prime}\right) \varsubsetneqq a^{*} b^{*} \mathcal{O}, \quad a^{\prime} \text { norm generators of } \mathscr{G}^{*} \text {. }
$$

Reduction Lemma 2.9. We may henceforth assume that

$$
\mathscr{D}\left(\delta \lambda_{\bar{u}} a^{\prime}\right)=a^{*} b^{*} \mathcal{O}
$$

for every $\bar{u} \in \mathfrak{M}_{u}$ and every norm generator $a^{\prime}$ of $\mathscr{G}^{*}$. (Of course, the same goes for all $\bar{v} \in \mathfrak{M}_{v}$ and all $a^{\prime}$ of $\mathscr{G}^{*}$.)

Proof. Since $u$ is a Type II vector so that, by definition, for each vector $\tilde{u} \in \mathfrak{M}_{u}$, the sublattice $M(u ; \tilde{u})$ has norm ideal equal to $a^{*} \mathcal{O}$. If there exists a vector $\bar{u} \in \mathfrak{M}_{u}$ with the property that

$$
\mathscr{D}\left(\delta \lambda_{\bar{u}} a^{*}\right) \varsubsetneqq a^{*} b^{*} \mathcal{O},
$$

then Corollary 2.8 together with a simple computation of the weight ideal $\mathscr{W}\left(\langle u\rangle^{\perp}\right)$ of $\langle u\rangle^{\perp}$ tell us that $\mathscr{W} M(u ; \bar{u})$ equals $b^{*} \mathcal{O}$. In other words, we have an equality of the norm groups

$$
\mathscr{G} M(u ; \bar{u})=\mathscr{G}^{*} \text {. }
$$

Now, pick any $\bar{v} \in \mathfrak{M}_{v}$ with $Q(\bar{v})=Q(\bar{u})$. Since $\lambda_{\bar{v}} \in \lambda_{\bar{u}} \mathscr{L}^{2}$, Corollary 2.8 implies that the norm group of $M(v ; \bar{v})$ must equal to $\mathscr{G}^{*}$ also. Hence,

$$
M(v ; \bar{v}) \cong M(u ; \bar{u})
$$

by Witt and O'Meara. Thus, $u \sim v$.

2.10. What Lemma 2.9 says, in effect, is that for any norm generator $a^{*}$ and weight generator $b^{*}$ of $\mathscr{G}^{*}$, and any vector $\bar{u} \in \mathfrak{M}_{u}$, the number $\delta$ has the special feature that 


$$
\delta \lambda_{\bar{u}}=a^{*} t^{2}+b^{*} \gamma
$$

where $\gamma$ is an unit. (Of course, $t$ is not an unit by Type II-ness.)

2.11. Proof of the Main Theorem for $\operatorname{dim} L=4$.

Proof. The following claims may be readily proved and we do not give the proofs here:

(I) For any binary nondepleted unimodular lattice $K$, whenever $K$ is represented as $K \cong A(\alpha, \gamma)$ either $\alpha$ or $\gamma$ must be a norm generator for $\mathscr{G} K$ and furthermore, the quadratic defect $\mathscr{D}(\alpha \gamma)$ must be $\mathscr{N} L \cdot \mathscr{W} L$.

(II) If $K$ has same hypothesis as in statement (I), and if, say, $\alpha$ is a norm generator for $\mathscr{G} K$ and $Q(x)=\alpha$, for some $x \in K$, then $K=\mathscr{O} x+\mathcal{O} y=A(\alpha, \beta)$-where one may take, if needed, $\beta$ to be a weight (base) generator.

Sublemma. Suppose $K \cong \mathscr{C}(a, b)$ with $a b \sim \pi$ and $b \notin 20^{\circ}-i n$ Riehm's notation, see [6]-and if $L$ is any binary unimodular lattice such that $F K$ (i.e., $F \otimes_{0} K$ ) is isometric to $F L$, and $a^{\prime} \in Q(L)$ is a norm generator for both $\mathscr{G} L$ and $\mathscr{G} K$, then $K \cong L$.

Proof. Let $Q(x)=a^{\prime}$, for some primitive vector $x \in L$. $F L$ isometric to $F K$ implies the discriminant of $K$ equals that of $L$ so that if we write the common discriminant as $-(1+a)$ whose defect is $\alpha \mathcal{O}$, then

$$
L \cong \mathscr{C}\left(a^{\prime},-\alpha a^{\prime-1}\right) .
$$

It is easy to see ord $\left(-\alpha a^{\prime-1}\right)=$ ord $(b)$. Hence, their weights (and therefore their norm groups as well) are equal.

Sublemma. There exists a vector $\bar{u} \in \mathfrak{M}_{u}$ such that $M(u ; \bar{u})$ has norm group equaling to $\mathscr{G}^{*}$.

Proof. Choose any $\widetilde{u} \in \mathfrak{M}_{u}$. If the norm group of $M(u ; \widetilde{u})$ is not $\mathscr{G}^{*}$, we put

$$
\langle u\rangle^{\perp} \cong M(u ; \widetilde{u}) \perp\left\langle\delta \lambda_{\tilde{u}}\right\rangle,
$$

where, since $L$ is 4 -dimensional $M(u ; \widetilde{u})$ is isometric to $A\left(a^{*}, \beta\right) . \quad(N$. $B$. We used Type II-ness here.) Performing an ou-transform (see [5]) changing $\beta$ to $\beta \perp \delta \lambda_{\tilde{u}}$ we see we endup with

$$
\langle u\rangle^{\perp} \cong M(u ; \bar{u}) \perp\left\langle\delta \lambda_{\bar{u}}\right\rangle,
$$

where 


$$
M(u ; \bar{u}) \cong A\left(a^{*}, \beta \perp \delta \lambda_{\tilde{u}}\right)
$$

whose norm group is obviously that of $\mathscr{G}^{*}$.

Using the results collected in this $\S 2.11$, the proof of the $\operatorname{dim} L=4$ case is apparent. Indeed, choose such a vector $\bar{u}$ in $\mathfrak{M}_{u}$ as mentioned in the sublemma. Choose a $\bar{v} \in \mathfrak{M}_{v}$ having $Q(\bar{u})=Q(\bar{v})$. Since 2.4 allows us to assume $\langle u\rangle^{\perp}$ is not depleted, one of the sublemmas implyies immediately that $M(u ; u) \cong M(v ; \bar{v})$.

2.12. Proof of the Main Theorem for $\operatorname{dim} L=5$. In view of Theorem 4.4, [2], it is sufficient to prove the following statement.

Proposition. Let $\operatorname{dim} E$ be either 5 or $6, u$ be a Type II vector in $L$ with $\langle u\rangle^{\perp}$ being nondepleted; and $Q(u)=\delta$ is a number satisfying the equation in $\S 2.10$. Then, there exists a vector $\bar{u} \in \mathfrak{M}_{u}$ such that $M(u ; \bar{u})$ is isotropic.

Proof. Recall $\mathscr{G}(\langle u\rangle)^{L}=\mathscr{C}^{*}=a^{*} \mathscr{O}^{2}+b^{*} \mathscr{O}$, for any norm generator $a^{*}$ and weight generator $b^{*}$ of $\mathscr{C}\left(\langle u\rangle^{\perp}\right)$. Pick any $\widetilde{u}$ from $\mathfrak{M}_{u}$. We see by $93: 21$, [4], the sublattice $M(u ; \widetilde{u})$ may be assumed to have norm group equal to $\mathscr{S}^{*}$.

If $\operatorname{dim} L=5$ then by $93: 18$, [4],

$$
M(u ; \widetilde{u}) \cong A\left(b^{\prime}, 4 \rho b^{\prime-1}\right) \perp\langle-d \Delta\rangle
$$

where $\Delta=1-4 \rho$. Here again, $b^{\prime}$ can be any weight generator! And $d$ is the discriminant of $M(u ; \widetilde{u})$. But, clearly by a suitable op-transformation on $M(u ; \widetilde{u})$, we can have the following splitting:

$$
M(u ; \tilde{u}) \cong A\left(b^{\prime} \perp(-d \Delta) s^{2}, 4 \rho b^{\prime-1}\right) \perp\langle\varepsilon\rangle
$$

for some unit $\varepsilon ; s$ can be any integer.

Now, $\S 2.10$ tells us that

$$
-\delta \lambda_{\tilde{u}}=(-d \Delta) t^{2}+\tilde{b},
$$

where $\tilde{b}$ is a weight generator and $t$ is not an unit because of Type II-ness. All we have to do next is to let $b^{\prime}$ to equal to $\widetilde{b}$ and apply the above op-transform so that $s=t$. Finally, apply another optransform on $\langle u\rangle^{\perp}$, this time, changing $b^{\prime} \perp(-d \Delta) s^{2}$ into

$$
b^{\prime} \perp(-d \Delta) s^{2} \perp \delta \lambda_{\tilde{u}}=0
$$

and the resulting picture looks like

$$
\langle u\rangle^{\perp} \cong A(0,0) \perp\langle\mu\rangle \perp\left\langle\delta \lambda_{\bar{u}}\right\rangle
$$


for some unit $\mu$ and some $\bar{u} \in \mathfrak{M}_{u}$. (N.B. We get surely an hyperbolic component because $4 \rho b^{\prime-1}$ lies in 20.) This is, of course, the vector $\bar{u}$ that does the job for us.

The proof of the case for $\operatorname{dim} L=6$ is almost identical except there the original sublattice $M(u ; \bar{u})$ looks like

$$
M(u ; \widetilde{u}) \cong A\left(b^{\prime}, 4 \rho b^{\prime-1}\right) \perp A\left(a^{\prime},[1-d \Delta] a^{\prime-1}\right)
$$

for arbitrarily chosen norm generator $a^{\prime}$ and weight generator $b^{\prime}$.

REMARK 2.13. An important observation to be made in the proof of the proposition in $\S 2.12$ is that under the conditions given in that proposition, one can always derive a vector $\bar{u} \in \mathfrak{M}_{u}$ such that the sublattice $M(u ; \bar{u})$ has an hyperbolic component. This is the key to the short proof the $\operatorname{dim} L=6$ case of our Main Theorem to be given below. Our first proof for this 6-dimensional situation involved long and elaborate arguments treating the vectors "case by case"; that is, considering them when they are both $\mathscr{N}-(\mathscr{G}-)$ regular, irregular, ... etc. Yet, it is precisely by looking at them at such detailed level that enabled us to realize the necessity for some result like our "Reduction Lemma", and hence the equality in $\S 2.10$.

\subsection{Proof of the Main Theorem for $\operatorname{dim} L=6$.}

Proof. By $\$ 2.13$, choose a vector $\bar{u} \in \mathfrak{M}_{u}$ such that $M(u ; \bar{u})$ incorporates an hyperbolic component. [N.B. Strictly speaking, the existence of such a vector $\bar{u} \in \mathfrak{M}_{u}$ has thus far been verified only when $M(u ; \bar{u})$ assumes the so-called " $K$-form", see $93: 18$, [4]; that is

$$
M(u ; \bar{u}) \cong A\left(b^{\prime}, 4 b^{\prime-1}\right) \perp A\left(a^{\prime},[1-d \Delta] \alpha^{\prime-1}\right) .
$$

It is not difficult to see, however, that if $M(u ; \bar{u})$ assumes the "J-form":

$$
M(u ; \bar{u}) \cong A\left(b^{\prime}, 0\right) \perp A\left(\alpha^{\prime},-\alpha \alpha^{\prime-1}\right)
$$

where $\alpha$ is that integer such that

$$
d=1+\alpha, \quad \mathscr{D}(d)=\alpha \mathcal{O},
$$

then, an entirely analogous argument carries through.]

A word of caution! The temptation here is to cancel the $A(0,0)$ component in both $\langle u\rangle^{\perp}$ and $\langle v\rangle^{\perp}$, and then claim a "reduction" to the quaternary case. The fallacy is clearly that the resulting characteristic sets in the now smaller lattices need not necessarily represent the same field elements any more! What one can claim instead is that one can indeed find a vector $u^{*}$ in $\mathfrak{M}_{u}$ such that $M\left(u ; u^{*}\right)$ has the "J-form" because 


$$
\begin{aligned}
\langle u\rangle^{\perp} & \cong A\left(a^{*}, \cdots\right) \perp A(0,0) \perp\left\langle\delta \lambda_{\bar{u}}\right\rangle \\
& \cong A\left(a^{*}, \cdots\right) \perp A\left(\delta \lambda_{\bar{u}}, 0\right) \perp\left\langle\delta \lambda_{u^{*}}\right\rangle \\
& \cong A\left(a^{*}, \cdots\right) \perp A\left(b^{*}, 0\right) \perp\left\langle\delta \lambda_{u^{*}}\right\rangle .
\end{aligned}
$$

for some $u^{*} \in \mathfrak{M}_{u}$

Pick any $v^{*}$ from $\mathfrak{M}_{v}$ such that $Q\left(v^{*}\right)$ equals $Q\left(u^{*}\right)$. Put

$$
\mathscr{G} M\left(v ; v^{*}\right)=a^{*} \mathscr{O}^{2}+\bar{b} \mathscr{O},
$$

for some $\bar{b} \in \mathcal{O}$. We now claim $M\left(v ; v^{*}\right)$ must also assume a " $J$-form" This is clear when $a^{*} \bar{b} \sim 1$. On the other hand, if $a^{*} \bar{b} \sim \pi$, then $b^{*} \bar{b} \sim 1$ so that since $F M\left(u ; u^{*}\right)$ is isometric to $F M\left(v ; v^{*}\right)$, our claim becomes clear. Therefore, we have

$$
M\left(v ; v^{*}\right) \cong A\left(a^{*}, \cdots\right) \perp A(\bar{b}, 0)
$$

and

$$
\langle v\rangle^{\perp} \cong M\left(v ; v^{*}\right) \perp\left\langle\delta \lambda_{v^{*}}\right\rangle .
$$

We are now presented in a situation which is strikingly similar to the 5-dimensional case in Theorem 4.4, [2]. Indeed, if $\bar{b}$ has order greater than that of $b^{*}$, a similar op-transformation finishes the proof.

2.15. The proof of the Main Theorem is now complete.

3. Effective computability.

3.1. Binary case. Given a maximal vector $u$ with quadratic length $Q(u)=\delta$, it is easy to find a vector $\bar{u}$ from $\mathfrak{M}_{u}$. Do the same for $\bar{v}$. Compute $Q(\bar{u})$ and $Q(\bar{v})$ and see if they are congruent modulo $\omega \mathcal{O}$, where $\omega$, as usual, denotes $\max \{\delta, 2\}$. If they are, then it is easily verified that $u$ and $v$ must be of the same parity so that Theorem 2.1 , [2], tells us $u \sim v$. If not, obviously $u$ and $v$ are not of the same parity. Hence, $u$ and $v$ are not integrally equivalent. Since the vectors $\bar{u}$ and $\bar{v}$ are arbitrarily chosen, we see the actual computation involved for checking integral equivalence in $\operatorname{dim} L=2$ is quite minimal.

3.2. Computationally, it is not always a pleasant task to determine $Q\left(\mathfrak{M}_{u}\right)$ for a given vector $u$. Fortunately, for sufficiently large dimension of the given lattice $L$, say $\operatorname{dim} L \geqq 5$, there is a good remedy. We have, indeed the following result.

THEOREM. Suppose one can find a single pair of vectors $\bar{u} \in \mathfrak{M}_{u}$ and $\bar{v} \in \mathfrak{M}_{v}$ such that $Q(\bar{u})=Q(\bar{v})$, and suppose further we have

$$
\mathscr{G}\left(\langle u\rangle^{\perp}\right)=\mathscr{G}\left(\langle v\rangle^{\perp}\right)=\mathscr{G}^{*},
$$


then, $u \sim v$ whenever $\operatorname{dim} L \geqq 5$.

Proof. Express

$$
\langle u\rangle^{\perp} \cong M(u ; \bar{u}) \perp\left\langle\delta \lambda_{\bar{u}}\right\rangle .
$$

By 93: 21, [4], there is a Jordan decomposition

$$
\langle u\rangle^{\perp} \cong M\left(u ; u^{*}\right) \perp\left\langle\delta \lambda_{u^{*}}\right\rangle
$$

such that $\lambda_{u^{*}}=\lambda_{\bar{u}}$, and $\mathscr{G}\left(M\left(u ; u^{*}\right)\right)=\mathscr{G}^{*}$. Do the same for vector $v$. We see then the norm groups for $M\left(u ; u^{*}\right)$ and $M\left(v ; v^{*}\right)$ are equal and moreover, $\langle u\rangle^{\perp} \cong\langle v\rangle^{\perp}$, whenever $u$ and $v$ are anisotropic vectors. If $\delta$ should be zero, then modulo radicals $\langle u\rangle^{\perp}$ is just $M(u ; \bar{u})$. Similarly for $\langle v\rangle^{\perp}$. But, $\mathscr{G}\left(\langle u\rangle^{\perp}\right)=\mathscr{G}(M(u ; \bar{u}))$ so that the hypothesis that the norm groups for $\langle u\rangle^{\perp}$ and $\langle v\rangle^{\perp}$ being equal implies here their isometry.

Suppose, for the moment, that $\operatorname{dim} L \geqq 7$, then $\operatorname{dim} M(u ; \bar{u}) \geqq 5$ so that

$$
Q(M(u ; \bar{u}))=\mathscr{G}(M(u ; \bar{u})) .
$$

Therefore, the hypothesis of the theorem here implies $Q\left(\mathfrak{M}_{u}\right)=Q\left(\mathfrak{M}_{v}\right)$ and $u \sim v$ by the Main Theorem.

For $\operatorname{dim} L=6$, again $M(u ; \bar{u})$ represents every element of its own norm group by a theorem of Riehm, see Theorem 7.4, [6]. So, once again $u \sim v$ by Main Theorem.

Let $\operatorname{dim} L=5$. If $u$ is $\mathscr{G}$-regular and $\delta$ satisfies condition (D) in the sense defined in $\S 3,[2]$, then it is not difficult to see with the help of Corollary 3.3, [2], that $u \sim v$. If $\delta$ does not satisfy condition (D), then, since we have already shown that $\langle u\rangle^{\perp} \cong\langle v\rangle^{\perp}$, we deduce $u \sim v$ by Proposition 3.4, [2]. So, let $u$ be $\mathscr{N}$-regular, but $\mathscr{G}$-irregular. If $\mathscr{D}\left(\delta \lambda_{\bar{u}} a^{*}\right) \varsubsetneqq a^{*} b^{*} \mathcal{O}=b^{*} \mathscr{O}$, then, we can show, by same argument in the proof of Main Theorem, that $\mathscr{G}(M(u ; \bar{u}))$ would equal to $\mathscr{G}^{*}$. Similarly, for $\bar{v}$ and $M(v ; \bar{v})$. Thus, $u \sim v$. If, on the other hand,

$$
\mathscr{D}\left(\delta \lambda_{\bar{u}} a^{*}\right)=b^{*} \mathcal{O},
$$

then, as in $\S 2.14$, we can find a vector $u^{*}$ such that $M\left(u ; u^{*}\right)$ supports an hyperbolic component and again we get

$$
Q\left(M\left(u ; u^{*}\right)\right)=\mathscr{G}\left(M\left(u ; u^{*}\right)\right)
$$

by Theorem 7.4, [6]. Everything repeats once more; $u \sim v$ is therefore clear.

The theorem is therefore proved. 
COROLlaRY 3.3. If $\operatorname{dim} L \geqq 6, \mathscr{G}\left(\langle u\rangle^{\perp}\right)$ equals $\mathscr{G}\left(\langle v\rangle^{\perp}\right)$ and there exists a single pair of vectors $\bar{u} \in \mathfrak{M}_{u}$ and $\bar{v} \in \mathfrak{M}_{v}$ such that

$$
Q(\bar{u}) \equiv Q(\bar{v}) \bmod \mathscr{C}\left(\langle u\rangle^{\perp}\right),
$$

then, $u \sim v$.

REMARK 3.4. As in the binary case, if one can concoct a single pair $\bar{u} \in \mathfrak{M}_{u}$ and $\bar{v} \in \mathfrak{M}_{v}$ such that $Q(\bar{u})$ is not congruent to $Q(\bar{v})$ modulo $\mathscr{G}^{*}$, then $u$ can not be integrally equivalent to $v$. Here again, therefore, the computation is reduced essentially to finding the norm groups for $\langle u\rangle^{\perp}$ and $\langle v\rangle^{\perp}$. The Jordan decompositions involved are rather simple and the associated fundamental invariants can usually be read off directly from an arbitrary Jordan splitting. In the cases for $\operatorname{dim} L=3$, 4 , it is expedient to check the classification of the given vectors $u$ and $v$ and then employ the results contained in $\S 3$, [2]. The exceptional cases in these dimensions must be handled via characteristic sets, which for such low dimensions are not computationally unmanageable.

\section{Cancellation theorems.}

One of the basic results in the study of integral quadratic forms over dyadic local rings is a result of O'Meara's which allows one to (orthogonally) cancel hyperbolic components. Over fields (characteristic not two), the classical Witt's Theorem can be stated in any of the two equivalent forms: the cancellation version and the extension version. The solutions given in this paper and in [2] completes the investigation of one-dimensional integral analogue of Witt Extension Theorem for the case of modular forms over any dyadic local ring. (N.B. Over rings, cancellation is not equivalent to extension.) At present, the theory of orthogonally cancelling equivalent forms over rings (even over dyadic local rings) is still practically nonexistent. In this short section, we observe some immediate consequences of our Main Result and others from $\S 3$.

Notation 4.1. If $T$ are $S$ and isometric sublattices of a given lattice $L$ and if $t \in T, s \in S$, then we write $t \sim s$ over $[T: S]$ to mean that there is an isometry $\sigma$ on $L$ such that $\sigma(t)=s$, and $\sigma(T)=S$.

THEOREM 4.2. Let $L=K_{u} \perp J_{u}=K_{v} \perp J_{v}$ with $J_{u} \cong J_{v}$ and $u \in K_{u}, v \in K_{v}$. Moreover,

$$
\begin{aligned}
& Q\left(J_{u}\right) \subseteq Q\left(\mathfrak{M}_{u} \text { in } K_{u}\right) \\
& Q\left(J_{v}\right) \subseteq Q\left(\mathfrak{M}_{v} \text { in } K_{v}\right)
\end{aligned}
$$

Then, $u \sim v$ over $L$ implies $u \sim v$ over $\left[K_{u}: K_{v}\right]$. 
COROLLARY 4.3. If $u$ is integrally equivalent to $v$ over

$$
K \perp A(0,0) \perp A(0,0)
$$

where $u$ and $v$ both lie in $K$, then $u \sim v$ over $K \perp A(0,0) . \quad(N . B$. Thus, if there are two hyperbolic components in the orthogonal complements of the vectors, one can always cancel at least one ef them.)

COROLlaRY 4.4. Let $L=K_{u} \perp J_{u}=K_{v} \perp J_{v}$ with $J_{u} \cong J_{v}$ and $u \in K_{u}, v \in K_{v}, \operatorname{dim} K_{u} \geqq 6$. Furthermore, $\mathscr{G} J_{u} \subseteq \mathscr{G}\left(\langle u\rangle^{\perp}\right.$ in $\left.K_{u}\right)$ and $\mathscr{G} J_{v} \subseteq \mathscr{G}\left(\langle v\rangle^{\perp}\right.$ in $\left.K_{v}\right)$. Then, $u \sim v$ over $L$ implies $u \sim v$ over $\left[K_{u}: K_{v}\right]$.

5. Characteristic two case. Although there is no longer the possibility for $L$ having dimension five, it is not difficult to see that the techniques introduced in the proof of the Main Theorem in $\$ 2$ carry through here in the characteristic two case-with obvious parallel arguments. Hence, the actual proofs are left as exercises to the readers. (Note, for example, that the case when both $u$ and $v$ are both Type I vectors is once more being taken care of by a result like that of Proposition 3.5, [2]. However, the proof for this proposition must be modified as follows. Pick any $\bar{u}$ from $\mathfrak{M}_{u}$. Let $\sigma$ be the isometry sending $\langle u\rangle^{\perp}$ onto $\langle v\rangle^{\perp}$. Put

$$
\sigma M(u, \bar{u})=M(v, \bar{v})
$$

for some $\bar{v} \in \mathfrak{M}_{v}$. If $\delta$ is integral, then the hypothesis of $u$ and $v$ being of the same parity implies the equality of norm groups

$$
\mathscr{C} L(u, \bar{u})=\mathscr{C} L(v, \bar{v}) .
$$

Hence, an isometry between the lattices $L(u, \bar{u})$ and $L(v, \bar{v})$ by theorems of Sah and Arf (the characteristic two parallels of O'Meara and Witt). If $\delta$ is not integral. Once again, define the space isometry:

$$
\Phi: F L(u, \bar{u}) \longrightarrow F L(v, \bar{v})
$$

sending $u$ onto $v$ and $u+\delta \bar{u}$ onto $\mu v+\delta \bar{v}$, where $\mu$ is that number in the ground field which appears from comparing the Arf invariants -instead of the discriminant comparisons as in the characteristic zero situation-of the two lattice. Namely,

$$
\delta Q(\bar{u})=\delta Q(\bar{v})+\mu^{2}+\mu .
$$

It is readily checked that $\Phi$ indeed is an lattice-isometry between $L(u, \bar{u})$ and $L(v, \bar{v})$.

Again, when $\delta$ is integral: after seeing $L(u, \bar{u})$ is isometric to 
$L(v, \bar{v})$. We apply Theorem $2.1,[2]$ whose characteristic two analogue was proved in [3].)

Thus, we state the result:

THEOREM. Let $L$ be any unimodular lattice over the local ring $\mathcal{O}=k[[\pi]]$ of formal power series in one uniformizing variable $\pi$ and $k$ being a finite field of characteristic two. Two maximal vectors having same quadratic lengths are integrally equivalent if and only if their respective characteristic sets represent the same elements in 0 . (N.B. The result is of course valid for any $\mathfrak{A}$-modular lattice, $\mathfrak{A}$ a fractional ideal in the quotient field of $\mathcal{O}^{\text {.) }}$

Clearly, the discussion about effective computability treads through a parallel course.

\section{REFERENCES}

1. J. S. Hsia, Integral equivalence for vectors over depleted modular lattices on dyadic local fields, Amer. J. Math. 90 (1968), 285-294.

2. Integral equivalence of vectors over local modular lattices, Pacific J. Math. 23 (1967), 527-542.

3. - A note on the integral equivalence of vectors in characteristic 2 , Math. Ann. 179 (1968), 63-69.

4. O. T. O'Meara, Introduction to quadratic forms, Grundlehren der Mathematischen Wissenschaften, Springer-Verlag, Berlin, 1963.

5. Integral equivalence of quadratic forms in ramified local fields, Amer. J. Math. 79 (1957), 157-186.

6. C. R. Riehm, On the integral representations of quadratic forms over local fields, Amer. J. Math. J. Math. 86 (1964), 25-62.

Received November 27, 1968. This work has been supported in part by the National Science Foundation under contract GP-8911.

The Ohio State University

Columbus, OHio 



\section{PACIFIC JOURNAL OF MATHEMATICS}

\section{EDITORS}

H. ROYDEN

Stanford University

Stanford, California

Richard Pierce

University of Washington

Seattle, Washington 98105
J. DUGUNDJI

Department of Mathematics

University of Southern California

Los Angeles, California 90007

BASIL GORDON

University of California

Los Angeles, California 90024

\section{ASSOCIATE EDITORS}
E. F. BECKENBACH
B. H. NEUMANN
F. WOLF
K. YosHIDA

\section{SUPPORTING INSTITUTIONS}

UNIVERSITY OF BRITISH COLUMBIA

CALIFORNIA INSTITUTE OF TECHNOLOGY

UNIVERSITY OF CALIFORNIA

MONTANA STATE UNIVERSITY

UNIVERSITY OF NEVADA

NEW MEXICO STATE UNIVERSITY

OREGON STATE UNIVERSITY

UNIVERSITY OF OREGON

OSAKA UNIVERSITY

UNIVERSITY OF SOUTHERN CALIFORNIA
STANFORD UNIVERSITY

UNIVERSITY OF TOKYO

UNIVERSITY OF UTAH

WASHINGTON STATE UNIVERSITY

UNIVERSITY OF WASHINGTON

*

AMERICAN MATHEMATICAL SOCIETY CHEVRON RESEARCH CORPORATION TRW SYSTEMS

NAVAL WEAPONS CENTER 


\section{Pacific Journal of Mathematics}

\section{Vol. 31, No. $1 \quad$ November, 1969}

James Burton Ax, Injective endomorphisms of varieties and schemes........

Richard Hindman Bouldin, A generalization of the Weinstein-Aronszajn

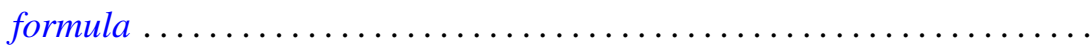

John Martin Chadam, The asymptotic behavior of the Klein-Gordon equation with external potential. II ...............................

Rina Hadass, On the zeros of the solutions of the differential equation

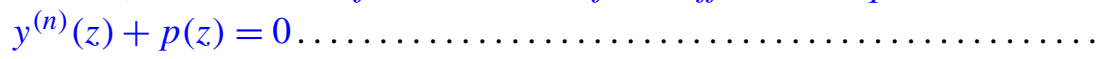

John Sollion Hsia, Integral equivalence of vectors over local modular lattices. II .............................................

Robert Hughes, Boundary behavior of random valued heat polynomial

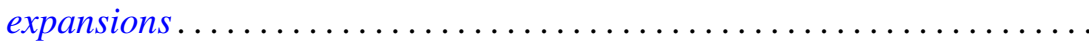

Surender Kumar Jain, Saad H. Mohamed and Surjeet Singh, Rings in which every right ideal is quasi-injective .........................

T. Kawata, On the inversion formula for the characteristic function .........

Erwin Kleinfeld, On right alternative rings without proper right ideals......

Robert Leroy Kruse and David Thomas Price, On the subring structure of

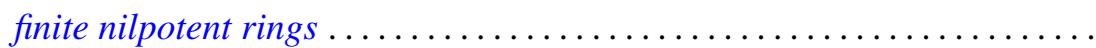

Marvin David Marcus and Stephen J. Pierce, Symmetric positive definite multilinear functionals with a given automorphism. .

William Schumacher Massey, Pontryagin squares in the Thom space of a bundle...

William Schumacher Massey, Proof of a conjecture of Whitney ...

John William Neuberger, Existence of a spectrum for nonlinear transformations

Stephen E. Newman, Measure algebras on idempotent semigroups ...

$\mathrm{K}$. Chandrasekhara Rao, Matrix transformations of some sequence spaces

Robert Bruce Schneider, Some theorems in Fourier analysis on symmetric

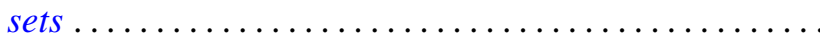

Ulrich F. K. Schoenwaelder, Centralizers of abelian, normal subgroups of hypercyclic groups...

Jerrold Norman Siegel, $G$-spaces, $H$-spaces and $W$-spaces

Robert Irving Soare, Cohesive sets and recursively enumerable Dedekind cuts...

Kwok-Wai Tam, Isometries of certain function spaces .... . .

Awadhesh Kumar Tiwary, Injective hulls of semi-simple modules over regular rings ....

Eldon Jon Vought, Concerning continua not separated by any nonaposyndetic subcontinuum .................... 